\title{
A rare complication of inguinal hernia repair: Total testicular ischemia and necrosis
}

\author{
Erhan Ates, Hakan Gorkem Kazici, Akin Soner Amasyali \\ Department of Urology Aydin Adnan Menderes University School of Medicine, Aydin, Turkey.
}

\begin{abstract}
Summary Testicular ischemia and necrosis are quite rare complications following inguinal hernia repair. There is still no consensus on the mechanism of infarction and necrosis in the literature. We present a case with total testicular ischemia and necrosis in the early period following the inguinal hernia repair with prolene mesh, ending up with orchiectomy.
\end{abstract}

KEY WORDS: Testis; Ischemia; Necrosis; Hernia repair.

Submitted 2 January 2018; Accepted 20 January 2018

\begin{abstract}
INTRODUCTION
Currently, prosthetic meshes are used commonly in inguinal hernia repair procedures as the duration of hospital stays are shorter, postoperative pain is less intense, and relapse rates are lower compared to the direct tissue to tissue repair surgeries (1). However, the direct contact of the mesh with the vessels in the inguinal canal or perimesh fibrosis can cause adverse effects on the testicular blood flow. These may result in some troublesome complications such as testicular atrophy, ischemic orchitis, a partial or total testicular ischemia and necrosis.

Testicular atrophies are observed at a rate of $0.5 \%$ after open inguinal hernioplasties, and the rate increases to $5 \%$ after recurrent hernioplasty surgeries, although the rates may vary depending on the experience level of surgeons (2).

We present a case with total testicular ischemia and necrosis in the early period following the inguinal hernia repair with prolene mesh, ending up with orchiectomy.
\end{abstract}

\section{Case presentation}

A 30-years-old male patient presented with pain and swelling in the left testis on the $5^{\text {th }}$ day following a herniorrhaphy with a prolene patch, in another healthcare facility, due to a strangulated hernia in the groin. Upon the consultation request of the emergency department, the patient was examined. The physical examination revealed that the left testis was slightly hard and tender and the spermatic cord was coarse on palpation. There was mild edema cutaneously and subcutaneously. The phren's sign was negative. Laboratory investigation results were $14.1 \mathrm{gr} / \mathrm{dL}$ for hemoglobin, $12700 \mathrm{mcL}$ for leukocytes, the platelet count was $288000 \mathrm{mcL}$, and
CRP was $93.4 \mathrm{mg} / \mathrm{L}$. The laboratory examination of the total urine revealed results within normal levels. During the ultrasound examination of scrotums, it was observed that the right testis was $29 \times 25 \times 44 \mathrm{~mm}$ in size with a normal blood supply, however, the left testis was larger $(40 \times 35 \times 52 \mathrm{~mm})$ with a decreased echogenicity compared to the right one. Doppler examination revealed that there is no blood supply to the left testis (Figure 1). In addition, there was some free fluid of about $2 \mathrm{~mm}$ height around the left testis.

Upon these findings, on the same day, the patient underwent left inguinal exploration in the region of the former incision scar. Following the exposure through the suture line towards the inguinal canal, a $100 \mathrm{cc}$ serous and hemorrhagic fluid was drained. The left spermatic cord was edematous. The left testis was dissected, reaching the level of the internal ring. The left spermatic cord was strangulated at the level of the internal ring by the mesh. At this level, an intracordal necrosis was present. Then, the tunica vaginalis was opened. The testis was necrotic (Figure 2).

The incision of the tunica albuginea did not result in arterial bleeding. Spermatic cord was clamped and a left orchiectomy was performed. The operation was finalized by placing a hemovac drain after revision of the mesh graft by a general surgeon. The hemovac drain was removed on the $2^{\text {nd }}$ day following the operation and the patient was discharged without any complications.

Figure 1.

Image of scrotal doppler ultrasonography. There is no blood flow to the left testis.

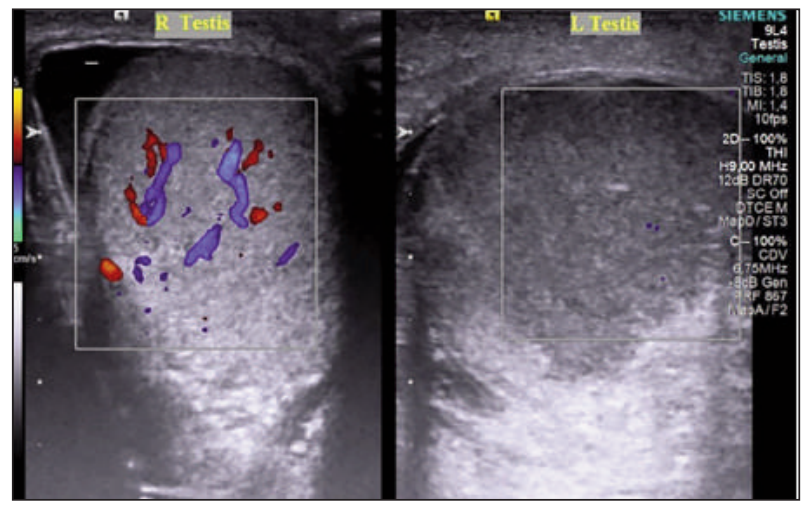

No conflict of interest declared. 


\section{Figure 2.}

Intraoperative images. Left testis and spermatic cord (A). Mechanical effect of prolene mesh (black arrow) on left spermatic cord (B), left necrotic testis (C).
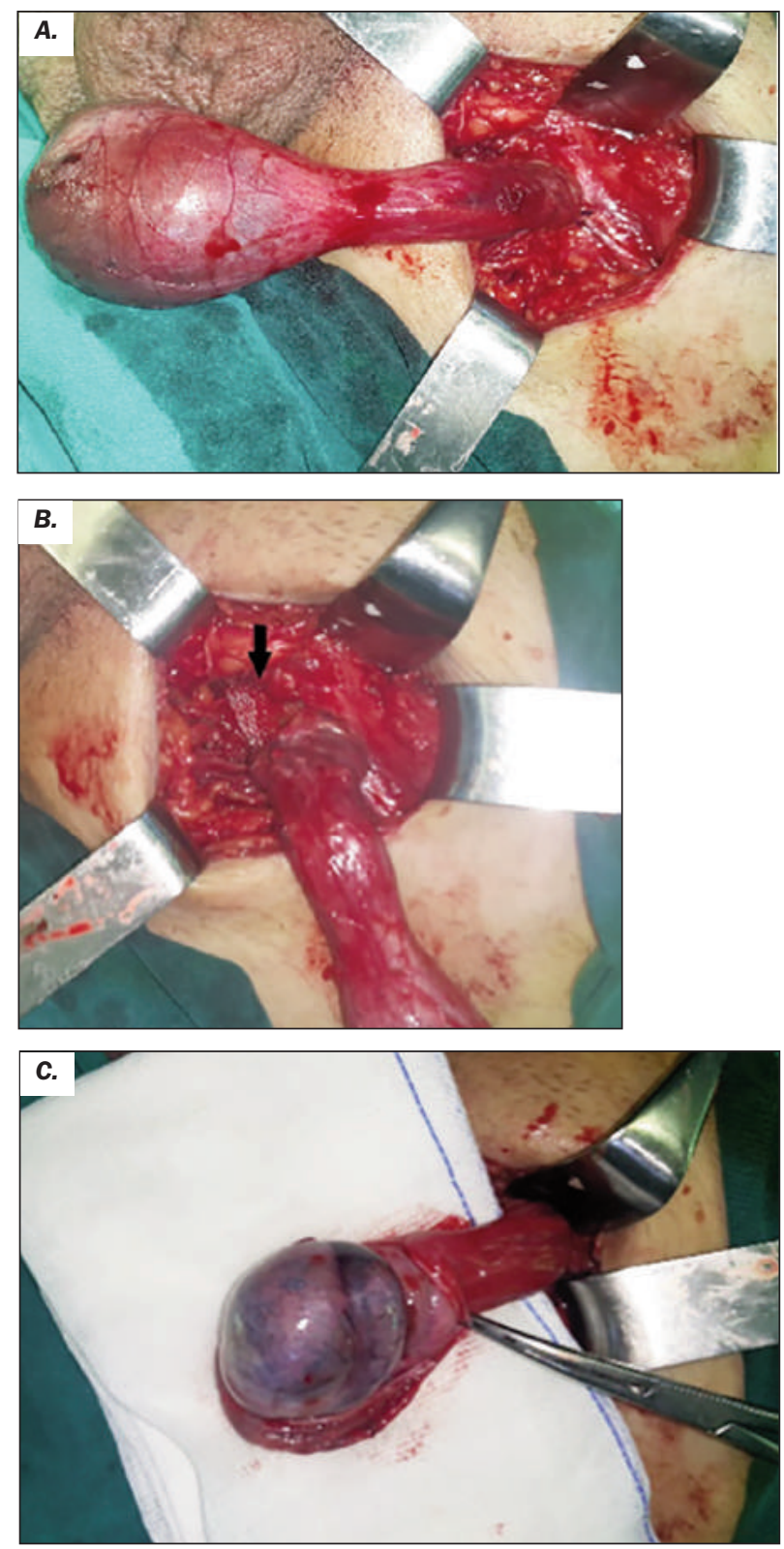

The patient presented with normal findings on the follow-up visit in the second postoperative week.

The pathological examination reported some testis tissue with disseminated ischemic necrosis and bleeding. Informed consent was taken from the patient.

\section{Discussion}

Testicular atrophy, ischemia, and necrosis are rare complications after inguinal hernia operations. While testicular atrophy is observed at a rate of $0.5 \%$ after primary open inguinal hernioplasty, the incidences may reach up to rates of $5 \%$ following open recurrent hernioplasties (2). This is considered to be due to an acute thrombosis of the pampiniform venous plexus rather than an acute artrial injury because of the collateral blood supply to the testis via the inferior epigastric, vesical, prostatic and scrotal arterial (3). Furthermore, there are studies reporting that testicular ischemia will not develop even, in onethird of the cases, in which the spermatic cord is ligated intentionally (4).

In a study, evaluating a total of 6500 patients, it has been determined that, following surgical traumas to the spermatic cord during inguinal hernioplasties, venous congestions develop, leading to thrombosis, which was considered to be the cause of consequent ischemic orchitis and testicular atrophies (5). Especially in large hernias extending to the scrotum and in recurrent hernias, wider dissections of the spermatic cord or dislocation of the testis from the scrotum during surgery increase the rates of ischemic orchitis and testicular atrophy. Therefore, it is argued that medial and inferior dissections extending beyond the pubic tubercle should be avoided during open repair surgeries (6).

The testicular veins may be affected mechanically as well as by inflammatory reactions due to prosthetic meshes. Peiper et al. (7), demonstrated inflammatory changes and testicular venous congestion following mesh implementations in animal studies. Inflammation was considered to be due to a fibrotic reaction, triggered by the mesh.

In Peiper's study, thrombosis in the spermatic cord was identified, additionally.

However, there is no consensus in the literature on the relation of the factors including the spermatic cord structures, testicular volumes, and changes in the arterial blood flow; to the direct contact of the prosthetic mesh or to perimesh fibrosis.

Uzzo et al. (8) observed decreases in the arterial perfusion of the testis and in its temperature. The ultrasonographic examination demonstrates decreases in the systolic blood flow and increases in the resistive index in testicular ischemia (9).

On the contrary, there are other studies arguing that testicular blood flow and perfusion does not change after hernia surgeries like the study by Zieren et al. (10).

Ischemic orchitis in the testis manifests on the $2^{\text {nd }}$ or $3^{\text {rd }}$ day following an inguinal hernia surgery and progresses to the develop an infarction.

The physical examination and Doppler ultrasonography are the initial diagnostic methods in cases, of which testicular ischemia is suspected (9). The testicular arterial blood flow and the testicular perfusion should be evaluated by a scrotal Doppler ultrasonography. In these cases, the possibility of a torsioned testis should be considered as well, despite the history of recent inguinal hernioplasty. In our case, the decision of exploration was made when it was determined that there was no blood supply to the testis.

The method of treatment is an emergency surgical intervention. Surgical methods vary depending on the observation of testicles during the surgery and on the duration of ischemia. The necrotic regions of the testis are excised and repaired by earlier surgical interventions, however, orchiectomy is inevitable when the early stage ischemia, following inguinal surgeries, is recognized later as it was in our case 


\section{Conclusions}

All kinds of symptoms such as scrotal pain, redness, and swelling should be evaluated immediately and with care after inguinal surgeries.

In addition, it must be considered that this type of complications may develop in any surgery adjacent to the spermatic cord.

\section{REFERENCES}

1. Huang CS, Huang CC, Lien HH. Prolene hernia system compared with mesh plug technique: a prospective study of short- to mid-term outcomes in primary groin hernia repair. Hernia 2005; 9:167-71.

2. Reid I, Devlin HB. Testicular atrophy as a consequence of inguinal hernia repair. The British journal of surgery 1994; 81:91-3.

3. Fong Y, Wantz GE. Prevention of ischemic orchitis during inguinal hernioplasty. Surg Gynecol Obstet, 1992; 174:399-402.

4. Heifetz CJ. Resection of the spermatic cord in selected inguinal hernias. Twenty years of experience. Arch Surg. 1971; 102:36-9.
5. Wantz GE. Testicular atrophy. A risk of inguinal hernioplasty. Chirurgie. 1991; 117:645-51.

6. Wantz GE. Open repair of hernias of the abdominal wall, surgical techniques. Sci Am Surg. 1995; 2:1-19.

7. Peiper $C$, Junge $K$, Klinge $U$, et al. Is there a risk of infertility after inguinal mesh repair? Experimental studies in the pig and rabbit. Hernia. 2006; 10:7-12.

8. Uzzo RG, Lemack GE, Morrissey KP, Goldstein M. The effects of mesh bioprothesis on the spermatic cord structures: A preliminary report in a canine model. J Urol. 1999; 161:1344-9.

9. Kupczyk-Joeris D, Kalb A, Hofer M, et al. Doppler sonography of testicular circulation following reconstruction of inguinal hernia. Chirurg. 1989; 60:536-40.

10. Zieren J, Beyersdorff D, Beier KM, Müller JM. Sexual function and testicular perfusion after inguinal hernia repair with mesh. Am J Surg. 2001; 181:204-6.

\section{Correspondence}

Erhan Ates, MD

drerhanates@yahoo.com

Hakan Gorkem Kazici, MD

hgkazici@yahoo.com

Akin Soner Amasyali, MD

drakinsoner@gmail.com

Department of Urology Aydin Adnan Menderes University School of Medicine, 09010 Aydin (Turkey) 